\title{
Repetitive Blast Exposure Produces White Matter Axon Damage without Subsequent Myelin Remodeling: In Vivo Analysis of Brain Injury Using Fluorescent Reporter Mice
}

\author{
Donald V. Bradshaw Jr, ${ }^{1,3}$ Yeonho Kim, ${ }^{2,3}$ Amanda Fu, ${ }^{2,3}$ Christina M. Marion, ${ }^{1,3}$ Kryslaine L. Radomski, ${ }^{2,3}$
} Joseph T. McCabe, ${ }^{1-3}$ and Regina C. Armstrong ${ }^{1-3, *}$

\begin{abstract}
The potential effects of blast exposure on the brain health of military personnel have raised concerns and led to increased surveillance of blast exposures. Neuroimaging studies have reported white matter abnormalities in brains of service members with a history of blast exposure. However, blast effects on white matter microstructure remain poorly understood. As a novel approach to screen for white matter effects, transgenic mice that express fluorescent reporters to sensitively detect axon damage and myelin remodeling were exposed to simulated repetitive blasts (once/day on 5 consecutive days). Axons were visualized using Thy 1-YFP-16 reporter mice that express yellow fluorescent protein (YFP) in a broad spectrum of neurons. Swelling along damaged axons forms varicosities that fill with YFP. The frequency and size of axonal varicosities were significantly increased in the corpus callosum (CC) and cingulum at 3 days after the final blast exposure, versus in sham procedures. CC immunolabeling for reactive astrocyte and microglial markers was also significantly increased. NG2CreER;mTmG mice were given tamoxifen (TMX) on days 2 and 3 after the final blast to induce fluorescent labeling of newly synthesized myelin membranes, indicating plasticity and/or repair. Myelin synthesis was not altered in the CC over the intervening 4 or 8 weeks after repetitive blast exposure. These experiments show the advantages of transgenic reporter mice for analysis of white matter injury that detects subtle, diffuse axon damage and the dynamic nature of myelin sheaths. These results show that repetitive low-level blast exposures produce infrequent but significant axon damage along with neuroinflammation in white matter.
\end{abstract}

Keywords: axon damage; blast exposure; myelin; transgenic reporter mice; traumatic brain injury; white matter

\section{Introduction}

Traumatic brain injury (TBI) is a significant public health concern for civilian and military populations. Brain injury associated with an explosive blast raises additional concerns due to the unknown effects of blast exposures and the potential for long-term symptoms. ${ }^{1,2}$ High explosives can result in injury from the blast wave along with result in injury to the head from projectiles striking the head or from the head hitting objects when the body is thrown. TBI associated with high explosive blasts accounted for approximately one-third of the nearly 400,000 military TBIs between 2001 and 2008. ${ }^{3}$ The effects of repetitive exposure to occupational blasts are an ongoing concern within the military. The National Defense Authorization Act (2018-2020) called for occupational surveillance to

${ }^{1}$ Graduate Program in Neuroscience, ${ }^{2}$ Center for Neuroscience and Regenerative Medicine, ${ }^{3}$ Department of Anatomy, Physiology, and Genetics, Uniformed Services University of the Health Sciences, Bethesda, Maryland, USA.

${ }^{4}$ Department of Neuroscience, Wexner Medical Center, Ohio State University, Columbus, Ohio, USA.

*Address correspondence to: Regina C. Armstrong, PhD, Department of Anatomy, Physiology, and Genetics, Uniformed Services University of the Health Sciences, 4301 Jones Bridge Road, Bethesda, MD 20814 USA; E-mail: Regina.armstrong@usuhs.edu

(c) Donald V. Bradshaw Jr et al., 2021; Published by Mary Ann Liebert, Inc. This Open Access article is distributed under the terms of the Creative Commons License (CC-BY) (http://creativecommons.org/licenses/by/4.0), which permits unrestricted use, distribution, and reproduction in any medium, provided the original work is properly credited. 
evaluate blast exposure, for example, among those engaged in breaching exercises or firing of heavy artillery. ${ }^{4}$ Early studies indicated a potential correlation between military personnel exposed to repeated lowlevel blast exposure and worsened long-term neurological and cognitive outcomes. ${ }^{5,6}$

The long-term effects of blast exposure on brain health remain poorly understood. Magnetic resonance imaging (MRI) studies using diffusion tensor imaging (DTI) detected changes in major white matter tracts of the brain in some deployed soldiers who experienced TBI with blast exposure. ${ }^{7-9}$ Studies of blast-exposed veterans with and without TBI found that blast exposure decreased DTI fractional anisotropy in several white matter tracts. ${ }^{10}$ Both breachers and veterans examined more than 1 year after blast exposure with TBI exhibited DTI changes in white matter. ${ }^{1,12}$ Further, veterans with blast-related mild TBI and loss of consciousness had increased white matter abnormalities and cognitive impairments. ${ }^{13}$

Post-mortem studies of brains of service members who experienced blast-related TBI have identified multiple neuropathological features. Axon damage, identified as axon swellings with accumulated $\beta$ amyloid precursor protein ( $\beta$-APP), has been observed along with disruptions to the myelin that ensheaths axons. ${ }^{14,15}$ Tau, a protein that is bound to microtubules in the cytoskeleton of healthy axons, has been reported to become hyperphosphorylated and form aggregates in neurons in the brains of some veterans who experienced repetitive head injury and blast exposure. ${ }^{16}$ Additionally, brains from military service members with a history of blast exposure exhibited astroglial scarring at interfaces between tissues of differing densities; this astrogliosis involved the subpial glial plate, penetrating cortical blood vessels, structures lining the ventricles, and white matter-gray matter junctions. ${ }^{17}$ Together, these neuroimaging and neuropathology studies indicate vulnerability of the brain to blast exposure and the potential for axons and white matter to be critical pathological factors. However, the full range of neuropathological effects of repetitive blast exposure is challenging to determine because potentially subtle pathological features may be sparsely distributed throughout the brain, and may change over time. To overcome these challenges, we evaluated the effects of blast exposure on axon and white matter pathology using two lines of transgenic mice with genetic reporter constructs that enable detection of in vivo changes in specific cellular processes.
Thy1-YFP-16 reporter mice express yellow fluorescent protein (YFP) in a broad spectrum of neurons so that individual axons can be visualized in diverse brain regions, including the corpus callosum (CC). ${ }^{18-21}$ We used Thy1-YFP-16 reporter mice to screen for individual damaged axons across the brain and brainstem. YFP-filled enlargements form at sites of damage and result in swelling and formation of varicosities along axons. The localization of YFP in axon swellings is a sensitive indicator of axonal injury ranging from small swellings observed early, when damage may still be reversible, through to intensely fluorescent terminal end bulbs of disconnected axons. ${ }^{22,23}$ Our previous work in Thy1-YFP-16 mice demonstrated YFP detection of traumatic axonal injury in white and gray matter regions of the brain at 3 days after single or repetitive concussive TBI. ${ }^{20,21}$ Importantly, in comparison with $\beta$-APP immunohistochemistry used for standard neuropathology, confocal analysis in Thy1YFP-16 mice can visualize YFP in three dimensions to identify terminal end bulbs at sites where axon damage has progressed to disconnection. ${ }^{24}$

Larger axons in white matter are wrapped by myelin sheaths that increase the efficiency and speed of action potential conduction. Myelin can be damaged by direct insults or as a secondary effect of axon damage. New myelin can be generated during activity-dependent adult plasticity and can form after injury or disease through regeneration and replacement of lost myelin sheaths. ${ }^{25-29}$ NG2CreER; $m$ TmG transgenic fluorescent reporter mice enable in vivo analysis of newly formed myelin sheaths over a specific time interval. ${ }^{21,30}$ The NG2 promoter is expressed in oligodendrocyte progenitor cells (OPCs) that proliferate and differentiate into myelin-forming oligodendrocytes. Tamoxifen (TMX) administration activates Cre recombinase that switches membrane fluorescent labeling from red to green in OPCs and their progeny. TMX given to NG2CreER; $m$ Tm $G$ mice on days 2 and 3 after TBI results in green fluorescent protein (GFP) labeling of newly generated oligodendrocytes and their myelin sheaths. ${ }^{21}$ We previously showed that a single concussive TBI increased formation of new myelin in the CC through 4 weeks, yet CC atrophy was evident when survival was extended to 8 weeks after TBI. ${ }^{21}$ In this study we leveraged the advantages of the NG2CreER;mTmG mice to examine new myelin formation over 4 and 8 weeks after repetitive low-level blast exposure.

The effect of blast waves on the brains of Thy1YFP-16 and NG2CreER;mTmG mice was tested using 
an Advanced Blast Simulator (ABS) to model the blast wave component of low-level exposures to explosives or heavy artillery relative to potential effects of repetitive blast exposures during deployment or training. Studies of service members have shown that experiencing three or more TBI events, with or without blast exposure, may increase effects compared with those with fewer or no TBI history. ${ }^{31,32}$ In mice, our prior studies of concussive mild repetitive TBI, with impacts once per day on each of 5 consecutive days, resulted in persistent neuroinflammation in the CC and adjacent cerebral cortex. ${ }^{20}$ Therefore, the current studies in transgenic reporter lines used repetitive low-level blast exposures given once per day on each of 5 consecutive days for analysis of axon damage, myelination, and neuroinflammation.

\section{Methods}

Mice

All mice were treated in accordance with guidelines of the Uniformed Services University of the Health Sciences (USUHS) and the National Institutes of Health Guide for the Care and Use of Laboratory Animals. Mice were socially housed as 2-4 mice per cage in $35 \mathrm{~cm} \times 16.5 \mathrm{~cm} \times 18 \mathrm{~cm}$ cages that contained enrichment objects and maintained a 12-h daytime light cycle (0600-1800) during which all experimental procedures were performed. The following mouse strains were obtained from the Jackson Laboratory (Bar Harbor, ME, USA): NG2CreER (RRID: IMSR_JAX:008538; B6.Cg-Tg(Cspg4-cre/Esr1*)BAkik/J), ROSA ${ }^{m T / m G}$ (RRID:IMSR_JAX:007676; B6.129(Cg)Gt(ROSA)26Sortm4(ACTB-tdTomato,-EGFP)Luo/J), and Thy1-YFP-16 (RRID:IMSR_JAX:003709; B6.Cg$\mathrm{Tg}($ Thy1-YFP)16Jrs/J). Mouse strains were bred as in-house colonies to generate experimental mice. Firstgeneration offspring from NG2CreER and $R O S A^{m T m G}$ breeders were used as NG2CreER;mTmG mice. The total number of Thy1-YFP-16 mice used was 15 ( 8 male, 7 female) and the total number of NG2CreER;mTmG mice used was 14 ( 10 male, 4 female). Male and female mice were combined for analyses after separate analyses showed no sex-based differences. Littermates of Thy1-YFP-16 mice or NG2CreER; $m$ TmG mice were used for quantitative analyses. The number of mice for each condition in each experiment is provided in each figure legend.

Repetitive blast procedures

At 8-9 weeks of age, mice were randomly assigned to repetitive sham (rSham) or repetitive blast (rBlast) pro- cedures. The rBlast mice were exposed to five consecutive simulated blast exposures, at 24-h intervals, delivered in an ABS (ORA, Inc., Fredericksburg, VA, USA). ABS procedures were performed with mice in the prone position for frontal blast exposure. Each mouse was anesthetized with $3 \%$ isoflurane and the head and body were secured to a wooden tongue depressor, wrapped in gauze, placed in mesh to prevent rotational movement, and positioned to face the oncoming blast as previously detailed. ${ }^{33,34}$ The rSham mice were anesthetized and the same procedures were followed except that the blast was not deployed. There was no significant difference in mean peak incident overpressure $(21.7 \pm 1.11 \mathrm{psi}, \sim 149.62 \mathrm{kPA})$, mean duration $(7.25 \pm 0.235 \mathrm{msec})$, or mean impulse (62.9 \pm 0.795 psi-msec) across the five blast exposures for any groups. Righting reflex time was significantly prolonged in rBlast mice $($ mean $=78.55 \mathrm{sec}$ ) compared with $\mathrm{rSham}$ mice $($ mean $=45.8 \mathrm{sec})$ (two-way analysis of variance [ANOVA]; $\mathrm{F}(1,28)=206.6 ; p>0.0001)$. Righting reflex time did not change significantly across the 5 days of rBlast or rSham procedures for either genotype or injury condition.

\section{Thy 1-YFP-16 mice: Analysis of axon damage}

At 3 days following the final rBlast or rSham procedure, Thy1-YFP-16 mice were perfused with $4 \%$ paraformaldehyde and brains were cut as $14-\mu$ m-thick cryosections. ${ }^{21}$ For qualitative analysis of the distribution of axon damage, sagittal sections of Thy1-YFP-16 mice were imaged using a Zeiss Axioscan Z.1 (Carl Zeiss) with a Plan-Apochromat $20 \times$ objective to image the entire brain section, from olfactory bulb to brainstem. To examine the three-dimensional (3D) structure of YFP-filled axons, confocal image stacks were acquired on a 700 laser scanning confocal microscope (Carl Zeiss) with a Plan-Apochromat $40 \times / 1.4$ oil or $63 \times /$ 1.4 oil objective and processed using Vision4D Modular Software (Arivis Vision4D, Phoenix, AZ, USA; RRID:SCR_018000).

Quantification of axonal varicosities focused on regions of interest (ROIs) in the CC and cingulum in coronal sections at levels between +0.5 and $-0.5 \mathrm{~mm}$ relative to bregma. The CC ROI extended from the midline bilaterally to the turning point of ventral descent in the external capsule. The cingulum ROI was evident as fibers oriented perpendicular to the underlying $\mathrm{CC}$ and distinct from the superior cortical tissue. Images acquired on an Olympus IX-70 fluorescence microscope with a SPOT RT3 camera (Diagnostic 
Instruments, Arnold, MD, USA) were analyzed using Image J software (National Institutes of Health, Bethesda, MD, USA; RRID: SCR_003070). The ROI area was measured with a $10 \times$ objective. Morphological measurements of YFP-filled swellings used images acquired with a $40 \times$ objective. The width of each axonal varicosity was measured in the plane transverse to the length of the axon. The width of CC axons was $<1.5 \mu \mathrm{m}$ as measured by electron microscopy. ${ }^{35,36}$ Pilot analyses in rSham and rBlast mice confirmed that axon diameter varied along normal-appearing axons but ranged from 0.5 to $1.5 \mu \mathrm{m}$. Therefore, quantification of axon damage included only those YFP-filled swellings $>2.5 \mu \mathrm{m}$ in width with peak fluorescent value (measured as maximum pixel intensity) of at least $25 \%$ greater than adjacent axons, which was above the $95 \%$ confidence interval of the YFP fluorescence intensity of adjacent normal-appearing axons. Cell bodies were distinguished using 4',6-diamidino-2-phenylindole (DAPI) nuclear staining. Axonal varicosities included YFP-filled swellings along an axon and terminal end bulbs, that is, swellings at the end of an axon segment. Analysis included six to eight sections per mouse.

Thy1-YFP-16 mice: analysis of neuroinflammation Mice perfused at 3 days following final rBlast or rSham procedures were further analyzed using immunohistochemistry in coronal sections at levels between +0.5 and $-0.5 \mathrm{~mm}$ relative to bregma. The CC ROI extended from the midline bilaterally to under the peak of the cingulum. ${ }^{21}$ Astrocytes were evaluated by immunostaining for glial fibrillary acidic protein (GFAP; polyclonal rabbit anti-GFAP; 1:1000; DAKO, Carpinteria, CA, USA; Agilent Cat\# N1506, RRID:AB 10013482). Microglia/macrophages were identified using polyclonal rabbit antibody against ionized calcium binding adaptor molecule 1(IBA1; 1:1000; Wako, Richmond, VA, USA; Cat\# 019-19741, RRID:AB_839504). All tissue sections were counterstained with DAPI nuclear stain (Sigma-Aldrich., St. Louis, MO, USA; D9542). For quantification, images within the CC ROI were acquired with a $10 \times$ objective on an Olympus IX-70 microscope using a SPOT RT3 camera. ImageJ software was used to threshold fluorescence levels to quantify the area of immunolabeling above background. ${ }^{28}$ Images were also acquired with a $40 \times$ objective to examine the morphology of IBA1 immunolabeled cells to compare the density of resting or activated cells. ${ }^{20,35,37}$ Analysis included six to eight sections per mouse.
NG2CreER;mTmG mice: analysis of myelin

Cohorts of NG2CreER; $m$ TmG littermates received rBlast or rSham procedures. TMX (10 mg in $20 \mathrm{mg} / \mathrm{mL}$ corn oil suspension; Millipore, Sigma-Aldrich; T5648) was administered via oral gavage on day 2 and 3 after the final blast or sham procedure. ${ }^{21,38}$ TMX induces Cre recombinase mediated deletion of a stop codon switches transcription from constitutive expression of membrane-localized tdTomato (mT) to NG2 driven expression of membrane-localized GFP (mG). Fluorescence was quantified in coronal sections at levels between +0.5 and $-0.5 \mathrm{~mm}$ relative to bregma. The CC ROI extended from the midline bilaterally to under the peak of the cingulum. ${ }^{21}$ Images were acquired on an Olympus IX-70 microscope using a SPOT RT3 camera. ImageJ software was used to threshold the signal to above background and quantify the area of membrane fluorescence from the CC ROI, as previously detailed for CC $\mathrm{mG}$ labeling and for immunolabeling of myelin. ${ }^{21,28}$ Confocal images were acquired with a $40 \times$ objective to examine the morphology of membranes labeled with $\mathrm{mG}$ and $\mathrm{mT}$. Additional tissue sections were immunostained for total myelin based on myelin oligodendrocyte glycoprotein (MOG; mouse monoclonal, 1:100; EMD Millipore, Burlington, MA, USA; mab5680; RRID: AB_1587278).

\section{Statistical analysis}

Mice were allocated to the rBlast or rSham procedures using the random number generator function in Microsoft Excel. Investigators were blinded to animal injury condition during tissue analysis until after completion of the data analysis. GraphPad Prism 8.0 software (RRID:SCR_002798) was used for statistical analysis and graphing. Bar graphs demonstrate means with standard error of the means (SEMs) as well as symbols for individual mouse values. Two-way ANOVA with repeated measures, for CC and cingulum within same subjects, and Sidak's post hoc multiple comparisons test were used for axonal varicosity comparisons. An unpaired Student's $t$ test was used to compare immunolabeling of astrocytes and microglia between groups. Two-way ANOVA with Sidak's post hoc multiple comparisons test was used at each time-point for comparing $\mathrm{mG}$ fluorescence and $\mathrm{CC}$ width between rSham and rBlast in NG2CreER;mTmG mice. Statistical significance was set at values of $p<0.05$. 


\section{Results}

Screening in Thy1-YFP-16 fluorescent reporter mice detects damaged axons following repetitive blast exposure

The effects of repetitive blast exposures on axons were examined in Thy1-YFP-16 fluorescent reporter mice that express YFP in diverse neuronal populations but in only a subset of those neurons, which allows visualization of individual axons (Fig. 1). Confocal microscopy identified YFP-filled axonal swellings, which can detect axonal injury ranging from early reversible damage through terminal axon disconnection. ${ }^{22,23}$ As an initial qualitative assessment, tissues were screened in parasagittal sections of the brain and brainstem at

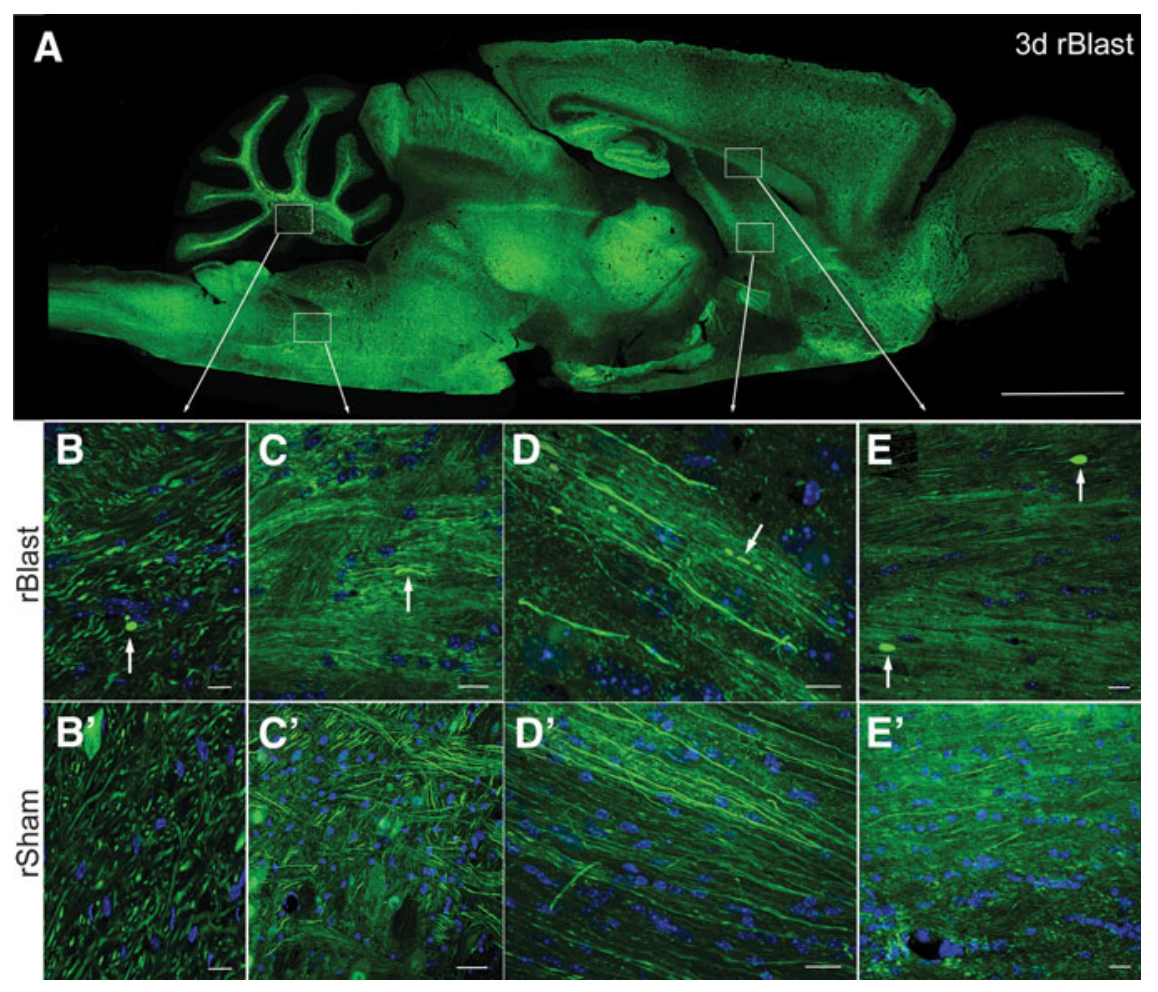

FIG. 1. Broad, sensitive screening for axon damage in brain sections after repetitive blast exposure using Thy1-YFP-16 mice. (A) Sagittal section from a Thy1-YFP-16 mouse at 3 days after rBlast exposure shows widely distributed neuron cell bodies and processes expressing yellow fluorescent protein (YFP; shown in green). (B-D') Sagittal high-magnification confocal microscope images of axons in white matter tracts in distributed brain regions of rBlast mice (B-D) and matching regions in rSham mice ( $\left.B^{\prime}-D^{\prime}\right)$. YFP shown in green. Nuclear stain 4',6-diamidino-2-phenylindole (DAPI) shown in blue. (B) Example of YFP accumulated in damaged axon to illustrate a terminal end bulb (arrow; transverse view) in the cerebellum of an rBlast mouse. Note that other YFP-filled oval and elongated structures are axons shown in angled cuts in both rBlast $(B)$ and rSham $\left(B^{\prime}\right)$ mice. The intense YFP of damaged axons is distinct from larger, more diffuse labeling in neuron cell bodies (adjacent to $B^{\prime}$ label). Examples from rBlast mice of YFP in an axonal thickening (arrow; longitudinal view) in the pons region of the brainstem $(C)$ and in fragmented axons (arrows; longitudinal view) in the column of the fornix (D). (E,E') Coronal confocal image showing corpus callosum axons in longitudinal view with two examples of intense YFP labeling in large terminal end bulbs in an rBlast section ( $E$; arrows), in comparison with an rSham section ( $\left.E^{\prime}\right)$. Low-power image $(A)$ is representative of images from three Thy1-YFP-16 mice examined in full sagittal sections. Higher-power images $\left(B-E^{\prime}\right)$ were acquired from sections of these or additional Thy1-YFP-16 mice examined in coronal sections for quantification in Figure 2. Scale bars $=2 \mathrm{~mm}(A), 20 \mu \mathrm{m}\left(B-E\right.$ and $\left.B^{\prime}-E^{\prime}\right)$. 
3 days after the last of five repetitive blast exposures (rBlast; Fig. 1A-E) that were delivered at 24-h intervals, or after matched procedures (rSham; Fig. 1B'-E') without deploying the blast. YFP-filled axonal swellings were observed in rBlast mice in diverse brain regions, including the cerebellum (Fig. 1B), pons (Fig. 1C), fornix (Fig. 1D), and CC (Fig. 1E). Overall, axons with YFP-filled swellings were found diffusely distributed in the brain of rBlast mice.

Repetitive blast exposure induces acute axon damage in white matter tracts

Further analysis of blast exposure in Thy1-YFP-16 mice focused on the CC and overlying cingulum, which had readily detected YFP-filled axonal varicosities in the rBlast mice (Fig. 1) and are clinically relevant areas of damage identified in human blast patients. ${ }^{10,17}$ These long white matter tracts also run perpendicular to one another, which is of interest relative to screening for broad effects of blast waves across brain regions. YFP-filled axonal varicosities were visualized at high resolution using confocal microscopy of coronal tissue sections through the $\mathrm{CC}$ and cingulum at 3 days after the final rBlast or rSham procedure (Fig. 2). The rSham mice showed YFP signal distributed within axons running longitudinally in the coronal plane or within interspersed crossing fibers (Fig. 2A). The rBlast mice exhibited distributed YFP signal in most axons but also rare axons with large accumulations of YFP (Fig. 2B). 3D reconstruction of confocal image stacks identified distinctly large axonal swellings to be terminal end bulbs. YFP accumulations were more frequent and often larger in rBlast mice relative to rSham mice (Fig. 2C-E).

\section{Repetitive blast exposure induces} neuroinflammation in the corpus callosum

To determine whether a neuroinflammatory response accompanied blast-induced axon pathology, coronal sections were immunolabeled for GFAP as a marker for astrocytes and IBA1 for microglia/macrophages in Thy1-YFP-16 mice at 3 days following final rBlast or rSham procedure (Fig. 3). The astrocytes and microglia have fine processes in the CC of rSham mice (Fig. 3A,B). Following rBlast exposure, reactive astrocytes and activated microglia exhibited shorter, thicker processes with increased immunoreactivity, indicative of a mild innate immune response (Fig. 3C,D). Quantitative analysis demonstrated a significant increase of immunoreactivity for GFAP and IBA1 in rBlast mice when compared with rSham (Fig. 3E,F). Further, rBlast exposure increased the density of IBA1 immunolabeled cells (Fig. 3G-I). These results provide evidence that rBlast exposures produce an acute neuroinflammatory response in the same tissue regions that exhibit axon damage.

FIG. 2. Repetitive blast exposure increases axon damage in the corpus callosum (CC) and adjacent cingulum. (A,B) Confocal images of coronal sections of the CC from Thy1-YFP-16 mice at 3 days after repetitive sham procedures ( $r$ Sham; $A$ ) or repetitive blast exposure ( $\mathrm{BBlast}$; $\mathrm{B}$ ) showing high magnification of yellow fluorescent protein signal (YFP) within axons and showing 4',6-diamidino-2-phenylindole (DAPI) nuclear stain in blue. The rSham mice exhibit relatively uniform YFP signal filling of axons viewed longitudinally (A). There are small variations in diameter along the length of the axons. In addition, YFP signal is visible in axons of crossing fibers cut transversely ( $A$; white arrow). An example in an rBlast mouse of an axon with a large, intensely fluorescent YFP-filled enlargement indicative of axon damage (B; red arrow) that is distinct from crossing fiber axons (B; white arrow). (C) Analysis of the frequency distribution indicates an increase in both the number and diameter of YFP accumulations in the CC and cingulum of rBlast mice compared with rSham controls. (D) The rBlast significantly increases the incidence of damaged axons with abnormal YFP localization (thickenings, varicosities, and terminal end bulbs) in the CC and cingulum, compared with rSham mice. (E) YFP accumulations in axonal varicosities are significantly larger in rBlast compared with rSham mice (B). Values are mean \pm standard error of the mean (SEM). Two-way analysis of variance (ANOVA) was used with repeated measures, for CC and cingulum within same subjects, and Sidak's post hoc multiple comparisons test. For the procedures, mouse numbers were: rSham group, $n=7$ and rBlast group, $n=8$. Scale bars $=20 \mu \mathrm{m}$ (A and B). 
Screening new myelin formation in NG2CreER;mTmG reporter mice following repetitive blast exposure

In addition to axon damage and neuroinflammation, white matter microstructural effects of blast detected by MRI could also involve myelin membranes that ensheath axons. ${ }^{14,39,40}$ We previously showed that NG2CreER;mTmG myelin reporter mice revealed an increase of new myelin synthesis in the CC over a 4-week period after single impact TBI, possibly indicating replacement of damaged myelin sheaths. ${ }^{21}$ Therefore, NG2CreER;mTmG mice were used to quantify new myelin synthesis following rBlast exposure (Fig. 4). TMX was administered on days 2 and 3 after the final blast or sham procedure, which corresponds with the timepoint for analysis of axon damage (Figs. 1-3).

Fluorescent green $\mathrm{mG}$ labeling identified new membranes synthesized after TMX induction. Clusters of
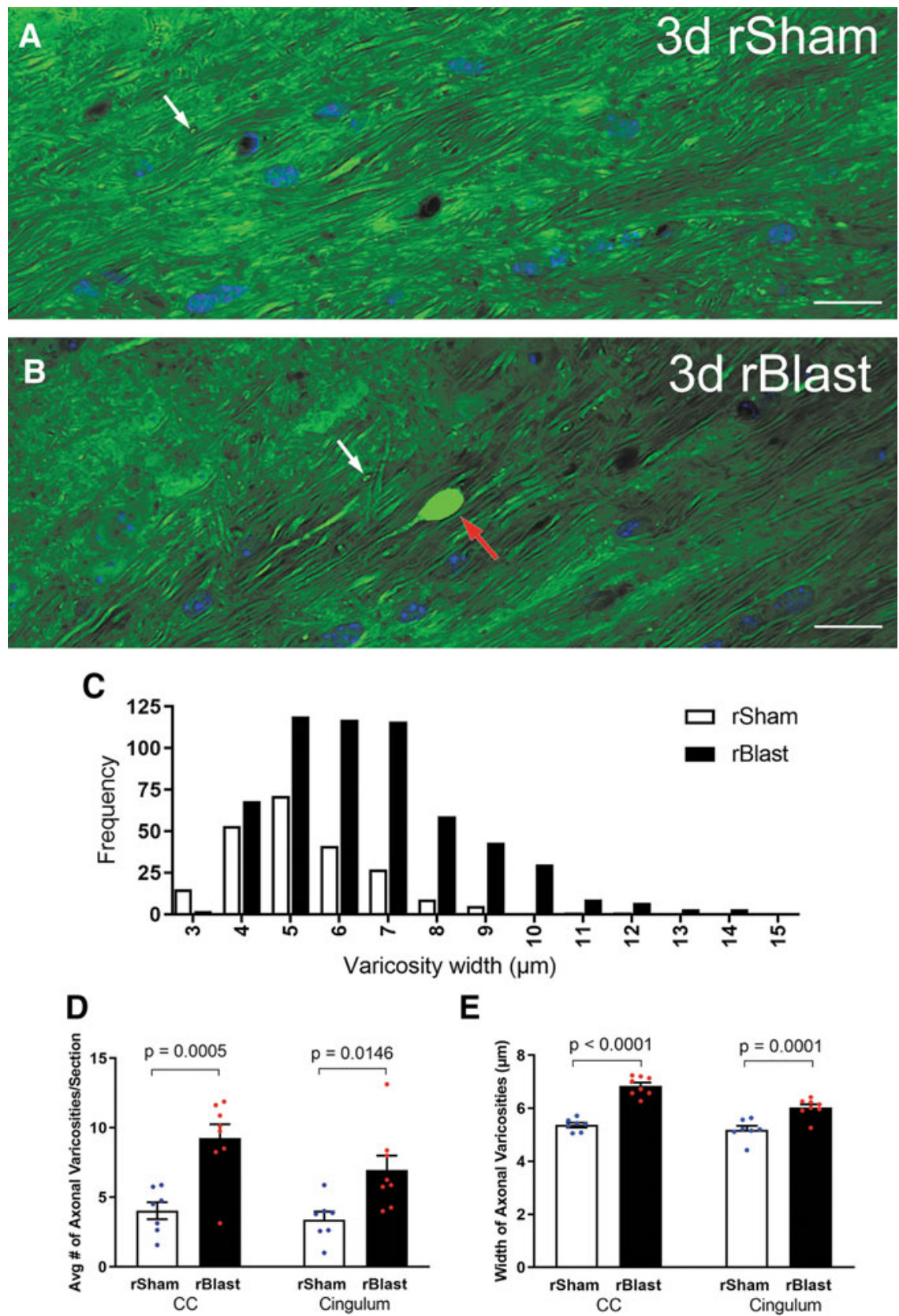

E

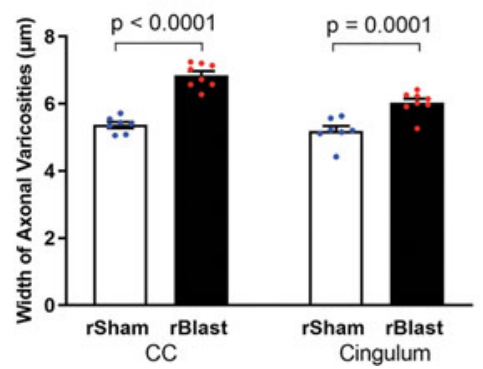



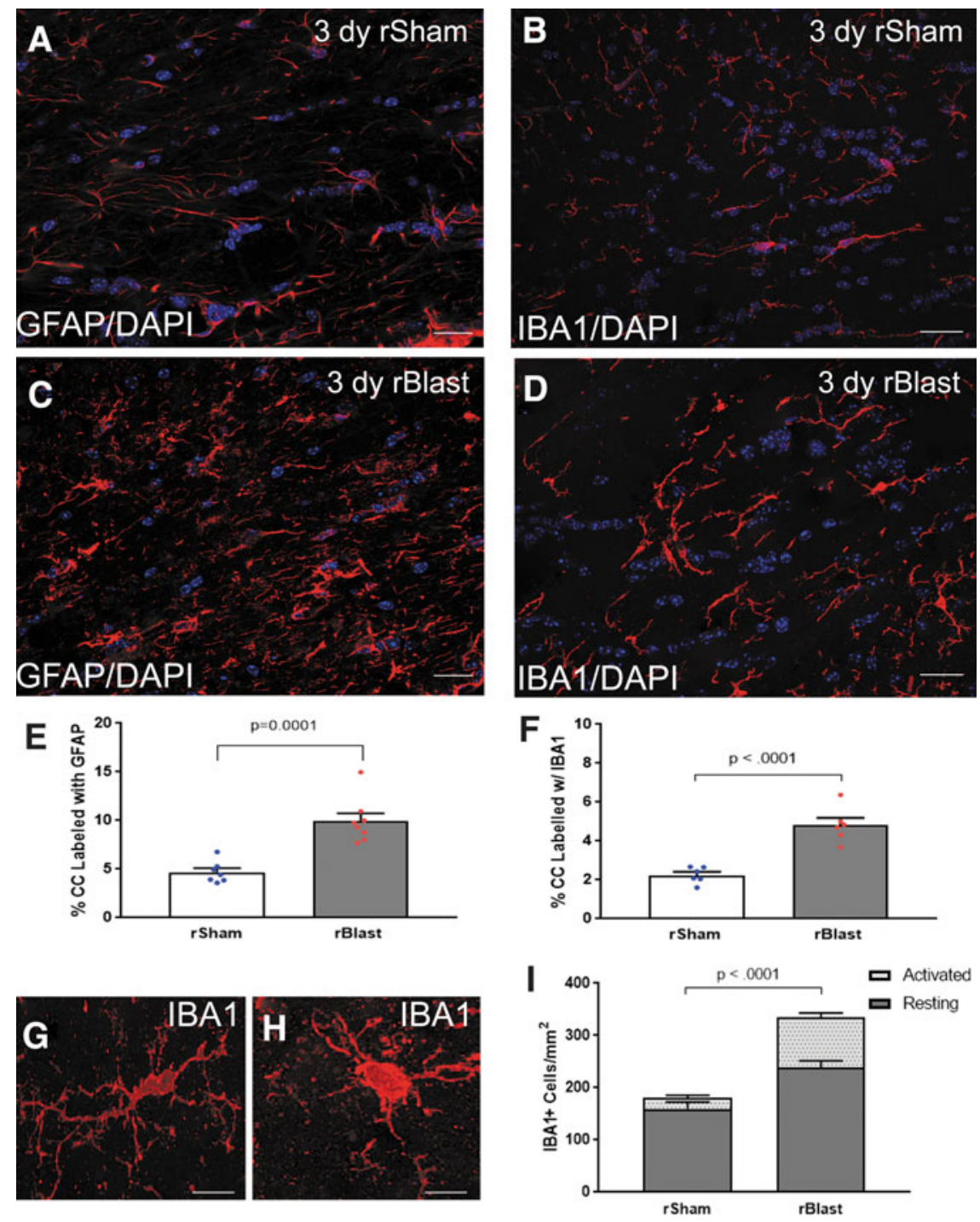

FIG. 3. Neuroinflammation is significantly increased in the corpus callosum (CC) after blast exposure. (A-D) Immunohistochemistry to detect glial fibrillary acidic protein (GFAP) in astrocytes and ionized calcium binding adaptor molecule 1 (IBA1) as a marker of microglia/macrophages. Confocal microscope images of coronal CC sections from Thy1-YFP-16 mice at 3 days after repetitive sham procedures (rSham; A,B) or repetitive blast exposure ( $r$ Blast; $C, D$ ). Astrocytes (pseudocolored white) and microglia/macrophages (red) have thicker processes with more intense immunoreactivity in rBlast mice as compared with rShams. Nuclear stain 4',6-diamidino-2-phenylindole (DAPI) shown in blue. (E,F) The rBlast exposure significantly increased immunoreactivity for GFAP (E) and IBA1 (F). (G,H) Representative confocal images showing IBA1+ cells as examples of resting state morphology with an elongated cell body and numerous processes $(\mathrm{G})$ or activated morphology with a more rounded and intensely immunolabeled cell body and processes (H). (I) rBlast exposure significantly increases the number of IBA1+ cells in the CC. Values are mean \pm standard error of the mean (SEM). Student's $t$ test was used to compare between groups. GFAP groups include rSham, $n=7$; rBlast, $n=8$. IBA1 groups include rSham, $n=6$; rBlast, $n=7$. Scale bars $=20 \mu \mathrm{m}$ $(\mathrm{A}-\mathrm{D})$ and $5 \mu \mathrm{m}(\mathrm{G}, \mathrm{H})$. 

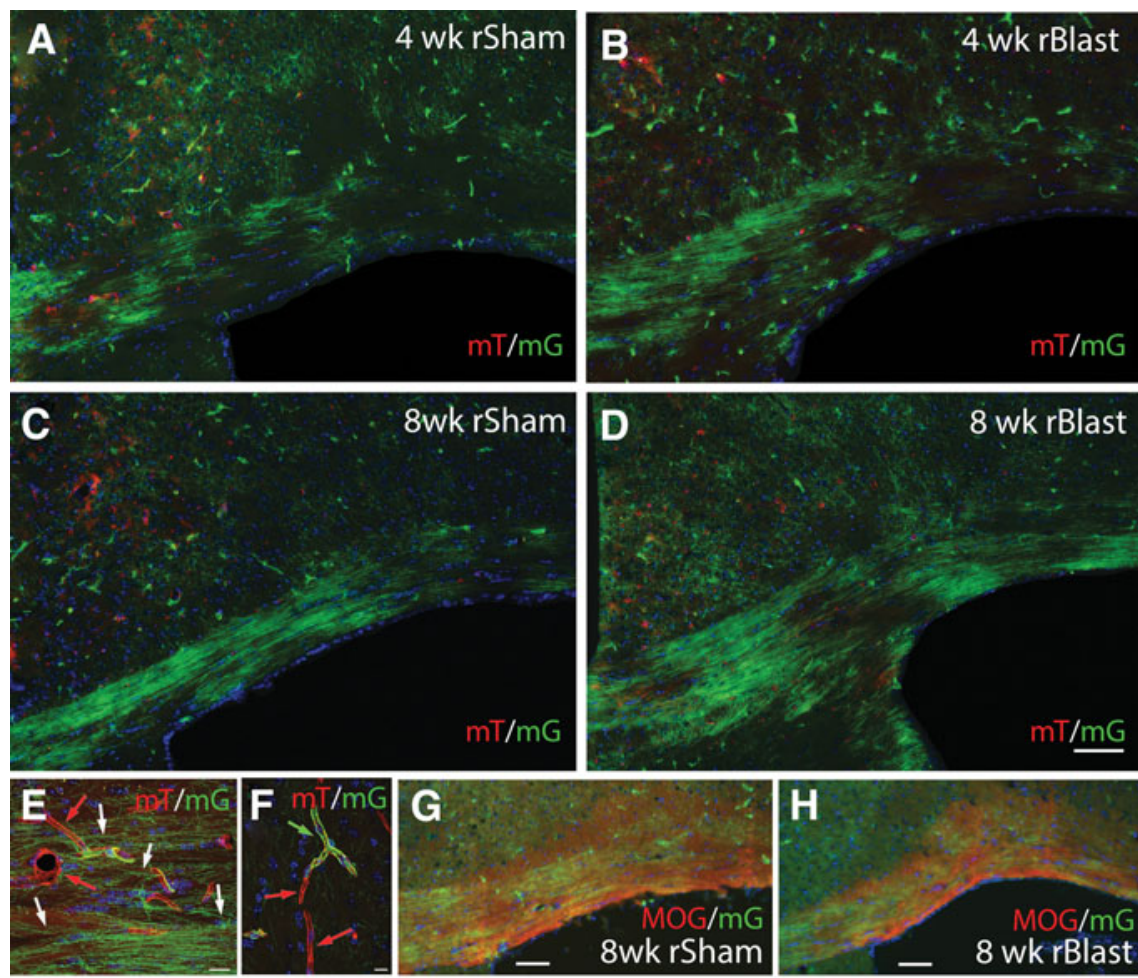

I
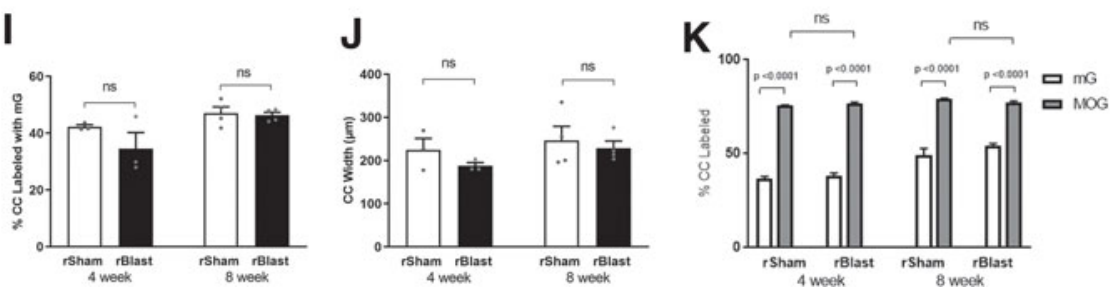

FIG. 4. Myelin membrane synthesis in the corpus callosum (CC) after repetitive blast exposure in $N G 2 C r e E R ; m T m G$ reporter mice. NG2CreER;mTmG mice were given tamoxifen (TMX) on days 2-3 after repetitive blast (rBlast) or sham (rSham) procedures. TMX induces recombination to stop expression of membrane-localized tdTomato $(\mathrm{mT})$ and initiate expression of membrane-localized green fluorescent protein $(\mathrm{mG})$ driven from the $\mathrm{NG} 2$ promoter (NG2mG). NG2 expression drives genetic $\mathrm{mG}$ fate-labeling of oligodendrocyte progenitors that is maintained in newly generated oligodendrocytes and their myelin membranes. (A-D) Representative images of $\mathrm{mG}$ (green) labeled membranes in coronal sections through the $C C$ at 4 weeks $(A, B)$ or 8 weeks $(C, D)$ after rSham $(A, C)$ or rBlast $(B, D)$. (E,F) High-magnification confocal microscope images within the $\mathrm{CC}(\mathrm{E})$ and cortex (F). Constitutive $\mathrm{mT}$ (red) labeling is visible in membranes of non-recombined cells, including blood vessels (E; red arrows). NG2 fate-labeled oligodendrocytes extend myelin-like membranes ( $E$; white arrows) along axons. NG2 fate-labeled pericyte ( $F$; green arrow) extend processes around blood vessels (F; red arrows). Nuclear stain 4',6-diamidino-2-phenylindole (DAPI) shown in blue. $(\mathbf{G}, \mathbf{H})$ Representative images of $\mathrm{mG}$ fluorescence (green) in newly synthesized myelin membranes along with immunolabeling for myelin oligodendrocyte glycoprotein (MOG) in new and persisting myelin membranes (red). The $\mathrm{mG}$ membranes represents only a subset of the total myelinated area of the CC. (I) rBlast did not significantly alter the proportion of mG-labeled myelin membranes synthesized in the CC at either time point. (J) rBlast exposure did not result in significant CC atrophy at either time-point.

(K) Quantification of $\mathrm{mG}$ labeling relative to total MOG immunolabeled myelin in the same sections. The myelinated fraction in the $\mathrm{CC}$ is significantly greater than the $\mathrm{mG}$ new myelin and is not changed by rBlast exposure. Values are mean \pm standard error of the mean (SEM). Two-way analysis of variance (ANOVA) with Sidak's post hoc multiple comparisons test. Four-week groups include rSham, $n=3$; rBlast, $n=3$. Eight-week groups include rSham, $n=4$; rBlast, $n=4$. Scale bars $=100 \mu \mathrm{m}(A-D$, shown in $D), 20 \mu \mathrm{m}(E-H)$. 
green myelin membranes were distributed in the CC at 4 weeks after the final $\mathrm{rBlast} / \mathrm{rSham}$ procedure (Fig. 4A,B) with further myelin formation by 8 weeks (Fig. 4C,D). These clusters are as expected with genetic fate-labeling of NG2 expressing progenitors that generate new oligodendrocytes, which each form myelin sheaths around many axons. Higher magnification showed green myelin aligned along axons (Fig. 4E). Green membranes extending along blood vessels are also occasionally observed due to NG2 expression in pericytes (Fig. 4F). Immunolabeling for MOG to detect myelin shows the presence of persisting myelin as well as newly synthesized $\mathrm{mG}$-labeled membranes (Fig. 4G,H). Quantification of $\mathrm{mG}$ membrane labeling in rSham mice showed substantial formation of new myelin membranes continues in adulthood (Fig. 4I). This findings is in agreement with studies that show CC myelination continues well beyond 16 weeks of age, ${ }^{36}$ which is the age match of 8 weeks post-blast. rBlast exposure did not change the extent of new myelin membrane synthesis in the CC after either 4 or 8 weeks (Fig. 4I). Further, in our prior study using NG2CreER;mTmG mice, single-impact TBI resulted in reduced CC width at 8 weeks, ${ }^{21}$ which was not found after rBlast exposure (Fig. 4J). Finally, rBlast exposures does not produce significant loss of CC myelin, as detected by MOG immunolabeling (Fig. 4K).

\section{Discussion}

The ability to detect subtle and distributed pathological features is vital to determining the effects of blast exposure on the brain. This study utilized two distinct fluorescent reporter mouse lines to assess components of potential axon and myelin white matter injury after rBlast exposures. The results demonstrate that rBlast exposure produces acute axon damage in multiple white matter tracts of the brain, as detected by screening in Thy1-YFP-16 mice. Acute axon damage was accompanied by an innate immune response of mildly reactive astrocytes and microglia. However, this white matter pathology did not lead to later effects on myelin synthesis, an indicator of myelin repair and remodeling, which was tracked in vivo after blast exposure using NG2CreER; $m$ TmG mice.

To model the blast wave component of low-level exposures to explosives or heavy artillery, the current experiments used an ABS device to generate controlled high-fidelity simulation of both positive and negative phases of "free-field" blast. ${ }^{33,41-44}$ Studies using singleblast exposures in the ABS in adult rats have produced long-term behavioral effects. ${ }^{44}$ Similar ABS exposures can alter the cerebral vasculature and the blood-brain barrier. ${ }^{41,42}$ Blast exposure can also alter cellular pathology including expression of markers for gliosis and myelinated axons (CNPase and neurofilament $\mathrm{H}$ ), but appears to vary with the degree of head immobilization. ${ }^{43}$ Indeed, single ABS exposure at 15 psi using a mesh to position the mouse in the prone position and minimize head movement resulted in a low-level blast frontal exposure that did not induce detectable microglial activation in the CC. ${ }^{33}$ The current studies used this same mesh procedure to similarly secure and position the mouse in the ABS. Our prior studies of concussive mild TBI in mice demonstrated persistent neuroinflammation in the CC and adjacent cortex after one impact per day on 5 consecutive days. ${ }^{20}$ With repetitive exposure pattern and increasing the ABS exposure to approximately $20 \mathrm{psi}$, we find a significant but low-level neuroinflammatory response in both astrocytes and microglia (Fig. 3). This CC neuroinflammation indicates a repetitive blast effect that warrants investigation of axon and myelin pathology.

Thy1-YFP-16 mice facilitated detection of axonal varicosities, including terminal end bulbs, which are significantly increased at 3 days after the final ABS exposure in the CC and cingulum (Figs. 1 and 2). Previous studies in rodent models of blast exposure found electrophysiological deficits and axon damage within white matter tracts along with behavioral deficits. ${ }^{45,46}$ Axon damage after blast exposure appears to be subtle and not readily detected by immunohistochemistry. ${ }^{47-49}$ The current YFP approach revealed individual damaged axons in mice even when exposed to a relatively low mean peak incident overpressure of approximately 20 psi (Fig. 2).

Our findings of acute neuroinflammation and axon damage in the CC led us to further examine myelination as an indicator of longer-term white matter injury after rBlast that is consistent with neuroimaging DTI findings in patients who have been experienced blasts. Myelin has been shown to be disrupted in TBI and myelin repair may have profound implications for recovery of axon function. ${ }^{50}$ Studies with open field explosive blasts reported CC pathology with increased silver staining of damaged axons and ultrastructural evidence of myelin disruption. ${ }^{40}$ However, this electron microscopic technique to evaluate myelin can only sample small regions. Additionally, the ability to monitor the dynamic turnover of myelin membranes is important to capture in evaluating potential 
myelin repair. In the current study, the use of NG2CreER;mTmG mice enabled quantification of in vivo myelin synthesis during specific intervals after blast exposures. During these periods, rBlast exposure did not produce late-phase effects on synthesis of new myelin membranes or induce CC atrophy (Fig. $4 \mathrm{G}, \mathrm{H}$ ). This result is in agreement with an overall mild level of white matter injury based on the limited degree of neuroinflammation and rare axon damage in the CC of rBlast mice. Possibly, TMX on days 2 and 3 could lessen blast pathology at 8 weeks. However, at 8 weeks after a concussive TBI, reduced CC width was found in two separate mouse studies in which one did not involve TMX and the other administered TMX using the current protocol. ${ }^{21,51}$

The advantages of mouse models to leverage the genetic labeling of cellular components are balanced by the limitations of small size and lack of complexity of the mouse brain. Mouse models are most useful as tools to screen for pathology that may be otherwise hard to distinguish. These findings in mice should facilitate further development of techniques applicable to higher mammals and humans, and interpretation of clinically applicable techniques such as white matter microstructure effects identified by MRI DTI. The devices available to model blast exposures increase reproducibility by reducing experimental variables and so do not fully replicate the human experience. In the broadest sense, the blast field overall is limited by the need for further studies to more fully establish the human neuropathology of TBI associated with blast exposure.

\section{Conclusion}

Evaluating effects of low-level repetitive blast exposure is challenging due to potentially subtle and diffuse pathology involving multiple cell types and tissues. Thy1YFP-16 mice detected axon damage in white matter tracts across multiple brain regions. NG2CreER;mTmG mice detected new myelin synthesis and provided the ability to visualize the neurovascular-pericyte unit. Taken together, this study demonstrates that fluorescent reporter mice are effective tools for evaluating cellular pathology of blast-induced neurotrauma and that repetitive low-level blast exposures produce infrequent but significant axon damage along with neuroinflammation in white matter.

\section{Acknowledgments}

The authors thank Dr. Genevieve Sullivan, Dr. Fengshan $\mathrm{Yu}$, Ms. Xiaomei Zi, and Mr. Tuan Le for technical assistance with this project. Opinions are those of the authors and do not represent the USUHS, the U.S. Department of Defense, or the federal government.

\section{Authors' Contributions}

Donald V. Bradshaw Jr wrote the first draft of the article, edited the article, designed and conducted experiments, and analyzed data. Yeonho Kim conducted blast exposures. Amanda Fu conducted blast exposures. Christina M. Marion performed research and edited the article. Kryslaine L. Radomski performed research and edited the article. Joseph McCabe contributed to the experimental design and edited the article. Regina C. Armstrong designed and performed research, analyzed data, and wrote and edited the article.

\section{Funding Information}

This research was supported by the U.S. Department of Defense at USUHS in the Partnership for Brain Injury and Disease Prevention, Treatment, and Research and in collaboration with the Center for Neuroscience and Regenerative Medicine Pre-clinical Core.

\section{Author Disclosure Statement}

No competing financial interests exist.

\section{References}

1. Mac Donald, C.L., Barber, J., Patterson, J., Johnson, A.M., Dikmen, S., Fann, J.R., and Temkin, N. (2019). Association between 5-year clinical outcome in patients with nonmedically evacuated mild blast traumatic brain injury and clinical measures collected within 7 days postinjury in combat. JAMA Netw. Open 2, e186676.

2. Aldag, M., Armstrong, R.C., Bandak, F., Bellgowan, P.S.F., Bentley, T., Biggerstaff, S., Caravelli, K., Cmarik, J., Crowder, A., DeGraba, T.J., Dittmer, T.A., Ellenbogen, R.G., Greene, C., Gupta, R.K., Hicks, R., Hoffman, S., Latta, R.C., 3rd, Leggieri, M.J. Jr., Marion, D., Mazzoli, R., McCrea, M., O'Donnell, J., Packer, M., Petro, J.B., Rasmussen, T.E., Sammons-Jackson, W., Shoge, R., Tepe, V., Tremaine, L.A., and Zheng, J. (2017). The biological basis of chronic traumatic encephalopathy following blast injury: a literature review. J. Neurotrauma 34, S26-S43.

3. Regasa, L.E., Agimi, Y., and Stout, K.C. (2019). Traumatic brain injury following military deployment: evaluation of diagnosis and cause of injury. J. Head Trauma Rehabil. 34, 21-29.

4. Engel, C.C., Hoch, E., Simmons, M.M. (2019). The neurological effects of repeated exposure to military occupational levels of blast: a review of scientific literature. RAND Conference Proceedings.

5. Carr, W., Stone, J.R., Walilko, T., Young, L.A., Snook, T.L., Paggi, M.E., Tsao, J.W., Jankosky, C.J., Parish, R.V., and Ahlers, S.T. (2016). Repeated low-level blast exposure: a descriptive human subjects study. Mil Med. 181, 28-39.

6. Tate, C.M., Wang, K.K., Eonta, S., Zhang, Y., Carr, W., Tortella, F.C., Hayes, R.L., and Kamimori, G.H. (2013). Serum brain biomarker level, neurocognitive performance, and self-reported symptom changes in soldiers repeatedly exposed to low-level blast: a breacher pilot study. J. Neurotrauma 30, 1620-1630.

7. Mac Donald, C.L., Barber, J., Andre, J., Panks, C., Zalewski, K., and Temkin, N. (2019). Longitudinal neuroimaging following combat 
concussion: sub-acute, 1 year and 5 years post-injury. Brain Commun. 1, fcz031.

8. Mac Donald, C.L., Johnson, A.M., Cooper, D., Nelson, E.C., Werner, N.J., Shimony, J.S., Snyder, A.Z., Raichle, M.E., Witherow, J.R., Fang, R., Flaherty, S.F., and Brody, D.L. (2011). Detection of blast-related traumatic brain injury in U.S. military personnel. N. Engl. J. Med. 364, 2091-2100.

9. Davenport, N.D., Lim, K.O., Armstrong, M.T., and Sponheim, S.R. (2012). Diffuse and spatially variable white matter disruptions are associated with blast-related mild traumatic brain injury. Neuroimage. 59, 2017-2024.

10. Ivanov, I., Fernandez, C., Mitsis, E.M., Dickstein, D.L., Wong, E., Tang, C.Y., Simantov, J., Bang, C., Moshier, E., Sano, M., Elder, G.A., and Hazlett, E.A. (2017). Blast exposure, white matter integrity, and cognitive function in Iraq and Afghanistan combat veterans. Front. Neurol. 8, 127.

11. Stone, J.R., Avants, B.B., Tustison, N.J., Wassermann, E.M., Gill, J., Polejaeva, E., Dell, K.C., Carr, W., Yarnell, A.M., LoPresti, M.L., Walker, P., O'Brien, M., Domeisen, N., Quick, A., Modica, C.M., Hughes, J.D., Haran, F.J. Goforth, C., and Ahlers, S.T. (2020). Functional and Structural Neuroimaging Correlates of Repetitive Low-Level Blast Exposure in Career Breachers. J. Neurotrauma 37, 2468-2481.

12. Taber, K.H., Hurley, R.A., Haswell, C.C., Rowland, J.A., Hurt, S.D., Lamar, C.D. and Morey, R.A. (2015). White matter compromise in veterans exposed to primary blast forces. J. Head Trauma Rehabil. 30, E15-E25.

13. Miller, D.R., Hayes, J.P., Lafleche, G., Salat, D.H., and Verfaellie, M. (2017). White matter abnormalities are associated with overall cognitive status in blast-related mTBI. Brain Imaging Behav. 11, 1129-1138.

14. McKee, A.C., and Robinson, M.E. (2014). Military-related traumatic brain injury and neurodegeneration. Alzheimers Dement. 10, S242-S253.

15. Ryu, J., Horkayne-Szakaly, I., Xu, L., Pletnikova, O., Leri, F., Eberhart, C., Troncoso, J.C., and Koliatsos, V.E. (2014). The problem of axonal injury in the brains of veterans with histories of blast exposure. Acta Neuropathol. Commun. 2, 153

16. Goldstein, L.E., Fisher, A.M., Tagge, C.A., Zhang, X.L., Velisek, L., Sullivan, J.A., Upreti, C., Kracht, J.M., Ericsson, M., Wojnarowicz, M.W., Goletiani, C.J., Maglakelidze, G.M., Casey, N., Moncaster, J.A., Minaeva, O., Moir, R.D., Nowinski, C.J., Stern, R.A., Cantu, R.C., Geiling, J., Blusztajn, J.K., Wolozin B.L., Ikezu, T., Stein, T.D., Budson, A.E., Kowall, N.W., Chargin, D., Sharon, A., Saman, S., Hall, G.F., Moss, W.C., Cleveland, R.O., Tanzi, R.E., Stanton, P.K., and McKee, A.C. (2012). Chronic traumatic encephalopathy in blastexposed military veterans and a blast neurotrauma mouse model. Sci. Transl. Med. 4, 134 ra60.

17. Shively, S.B., Horkayne-Szakaly, I., Jones, R.V., Kelly, J.P., Armstrong, R.C., and Perl, D.P. (2016). Characterisation of interface astroglial scarring in the human brain after blast exposure: a post-mortem case series. Lancet Neurol. 15, 944-953.

18. Feng, G., Mellor, R.H., Bernstein, M., Keller-Peck, C., Nguyen, Q.T., Wallace, M., Nerbonne, J.M., Lichtman, J.W., and Sanes, J.R. (2000). Imaging neuronal subsets in transgenic mice expressing multiple spectral variants of GFP. Neuron 28, 41-51.

19. Xie, M., Tobin, J.E., Budde, M.D., Chen, C.-I., Trinkaus, K., Cross, A.H., McDaniel, D.P., Song, S.-K., and Armstrong, R.C. (2010). Rostrocaudal analysis of corpus callosum demyelination and axon damage across disease stages refines diffusion tensor imaging correlations with pathological features. J. Neuropathol. Exp. Neurol. 69, 704-716.

20. Yu, F., Shukla, D.K., Armstrong, R.C., Marion, C.M., Radomski, K.L., Selwyn, R.G., and Dardzinski, B.J. (2017). Repetitive model of mild traumatic brain injury produces cortical abnormalities detectable by magnetic resonance diffusion imaging, histopathology, and behavior. J. Neurotrauma 34, 1364-1381.

21. Marion, C.M., Radomski, K.L., Cramer, N.P., Galdzicki, Z., and Armstrong R.C. (2018). Experimental traumatic brain injury identifies distinct early and late phase axonal conduction deficits of white matter pathophysiology, and reveals intervening recovery. J. Neurosci. 38, 87238736.

22. Greer, J.E., Hånell, A., McGinn, M.J., and Povlishock, J.T. (2013). Mild traumatic brain injury in the mouse induces axotomy primarily within the axon initial segment. Acta Neuropathologica 126, 59-74.

23. Gu, Y., Jukkola, P., Wang, Q., Esparza, T., Zhao, Y., Brody, D., and Gu, C. (2017). Polarity of varicosity initiation in central neuron mechanosensation. J. Cell Biol. 216, 2179-2199.

24. Weber, M.T., Arena, J.D., Xiao, R., Wolf, J.A., and Johnson, V.E. (2019). CLARITY reveals a more protracted temporal course of axon swelling and disconnection than previously described following traumatic brain injury. Brain Pathol. 29, 437-450.

25. McKenzie, I.A., Ohayon, D., Li, H., Paes de Faria, J., Emery, B., Tohyama, K. and Richardson, W.D. (2014). Motor skill learning requires active central myelination. Science 346, 318-322.

26. Monje, M. (2018). Myelin plasticity and nervous system function. Ann. Rev Neurosci. 41, 61-76.

27. Ortiz, F.C., Habermacher, C., Graciarena, M., Houry, P.Y., Nishiyama, A., Nait Oumesmar, B., and Angulo, M.C. (2019). Neuronal activity in vivo enhances functional myelin repair. JCl Insight 5, e123434.

28. Armstrong, R.C., Le, T.Q., Flint, N.C., Vana, A.C., and Zhou, Y.-X. (2006). Endogenous cell repair of chronic demyelination. J. Neuropathol. Exp. Neurol. 65, 245-256.

29. Sanchez, M.A., and Armstrong, R.C. (2018). Postnatal Sonic hedgehog (Shh) responsive cells give rise to oligodendrocyte lineage cells during myelination and in adulthood contribute to remyelination. Exp. Neurol. 299, 122-136.

30. Powers, B.E., Sellers, D.L., Lovelett, E.A., Cheung, W., Aalami, S.P., Zapertov, N., Maris, D.O., and Horner, P.J. (2013). Remyelination reporter reveals prolonged refinement of spontaneously regenerated myelin. Proc. Natl. Acad. Sci. U S A 110, 4075-4080.

31. Olivera, A., Lejbman, N., Jeromin, A., French, L.M., Kim, H.S., Cashion, A. Mysliwiec, V., Diaz-Arrastia, R., and Gill, J. (2015). Peripheral total tau in military personnel who sustain traumatic brain injuries during deployment. JAMA Neurol. 72, 1109-1116.

32. Cook, P.A., Johnson, T.M., Martin, S.G., Gehrman, P.R., Bhatnagar, S., and Gee, J.C. (2018). A retrospective study of predictors of return to duty versus medical retirement in an active duty military population with blast-related mild traumatic brain injury. J. Neurotrauma 35 991-1002.

33. Vu, P.A., Tucker, L.B., Liu, J., McNamara, E.H., Tran, T., Fu, A.H., Kim, Y., and McCabe, J.T. (2018). Transient disruption of mouse home cage activities and assessment of orexin immunoreactivity following concussive- or blast-induced brain injury. Brain Res. 1700, 138-151.

34. Jaiswal, S., Knutsen, A.K., Wilson, C.M., Fu, A.H., Tucker, L.B., Kim, Y., Bittner, K.C., Whiting, M.D., McCabe, J.T., and Dardzinski, B.J. (2019). Mild traumatic brain injury induced by primary blast overpressure produces dynamic regional changes in [(18)F]FDG uptake. Brain Res. 1723, 146400

35. Mierzwa, A.J., Marion, C.M., Sullivan, G.M., McDaniel, D.P., and Armstrong, R.C. (2015). Components of myelin damage and repair in the progression of white matter pathology after mild traumatic brain injury. J. Neuropathol. Exp. Neurol. 74, 218-232.

36. Sturrock, R.R. (1980). Myelination of the mouse corpus callosum Neuropathol. Appl. Neurobiol. 6, 415-420.

37. Sullivan, G.M., Mierzwa, A.J., Kijpaisalratana, N., Tang, H., Wang, Y., Song S.K., Selwyn, R., and Armstrong, R.C. (2013). Oligodendrocyte lineage and subventricular zone response to traumatic axonal injury in the corpus callosum. J. Neuropathol. Exp. Neurol. 72, 1106-1125.

38. Mierzwa, A.J., Sullivan, G.M., Beer, L.A., Ahn, S., and Armstrong, R.C. (2014) Comparison of cortical and white matter traumatic brain injury models reveals differential effects in the subventricular zone and divergent Sonic hedgehog signaling pathways in neuroblasts and oligodendrocyte progenitors. ASN Neuro. 6, 1759091414551782.

39. Petrie, E.C., Cross, D.J., Yarnykh, V.L., Richards, T., Martin, N.M., Pagulayan K., Hoff, D., Hart, K., Mayer, C., Tarabochia, M., Raskind, M.A., Minoshima, S., and Peskind, E.R. (2014). Neuroimaging, behavioral, and psychological sequelae of repetitive combined blast/impact mild traumatic brain injury in Iraq and Afghanistan war veterans. J. Neurotrauma 31, 425-436

40. Spees, W.M., Lin, T.-H., Sun, P., Song, C., George, A., Gary, S.E., Yang, H.-C., and Song, S.-K. (2018). MRI-based assessment of function and dysfunction in myelinated axons. Proc. Natl. Acad. Sci. U S A 115, E10225E10234.

41. Heyburn, L., Abutarboush, R., Goodrich, S., Urioste, R., Batuure, A., Statz, J., Wilder, D., Ahlers, S.T., Long, J.B., and Sajja, V. (2019). Repeated lowlevel blast overpressure leads to endovascular disruption and alterations in TDP-43 and Piezo2 in a rat model of blast TBI. Front. Neurol. 10, 766.

42. Rodriguez, U.A., Zeng, Y., Parsley, M.A., Hawkins, B.E., Prough, D.S., and DeWitt, D.S. (2019). Effects of blast-induced neurotrauma on pressurized rodent middle cerebral arteries. J. Vis. Exp. DOI: 10.3791/58792. 
43. Sawyer, T.W., Wang, Y., Ritzel, D.V., Josey, T., Villanueva, M., Shei, Y., Nelson, P., Hennes, G., Weiss, T., Vair, C., Fan, C., and Barnes, J. (2016). High-fidelity simulation of primary blast: direct effects on the head. J. Neurotrauma 33, 1181-1193.

44. Arun, P., Wilder, D.M., Eken, O., Urioste, R., Batuure, A., Sajja, S., Van Albert, S., Wang, Y., Gist, I.D., and Long, J.B. (2020). Long-term effects of blast exposure: a functional study in rats using an advanced blast simulator. J. Neurotrauma 37, 647-655.

45. Park, E., Eisen, R., Kinio, A., and Baker, A.J. (2013). Electrophysiological white matter dysfunction and association with neurobehavioral deficits following low-level primary blast trauma. Neurobiol. Dis. 52, 150-159.

46. Yin, T.C., Voorhees, J.R., Genova, R.M., Davis, K.C., Madison, A.M., Britt, J.K., Cintron-Perez, C.J., McDaniel, L., Harper, M.M., and Pieper, A.A. (2016). Acute axonal degeneration drives development of cognitive, motor, and visual deficits after blast-mediated traumatic brain injury in mice. eNeuro 3, ENEURO.0220-16.2016.

47. Garman, R.H., Jenkins, L.W., Switzer, R.C. 3rd, Bauman, R.A., Tong, L.C., Swauger, P.V., Parks, S.A., Ritzel, D.V., Dixon, C.E., Clark, R.S., Bayir, H., Kagan, V., Jackson, E.K., and Kochanek, P.M. (2011). Blast exposure in rats with body shielding is characterized primarily by diffuse axonal injury. J. Neurotrauma 28, 947-959.

48. Koliatsos, V.E., Cernak, I., Xu, L., Song, Y., Savonenko, A., Crain, B.J., Eberhart, C.G., Frangakis, C.E., Melnikova, T., Kim, H., and Lee, D. (2011). A mouse model of blast injury to brain: initial pathological, neuropathological, and behavioral characterization. J. Neuropathol. Exp. Neurol. 70, 399-416.

49. Murphy, E.K., lacono, D., Pan, H., Grimes, J.B., Parks, S., Raiciulescu, S., Leonessa, F., and Perl, D.P. (2020). Explosive-driven double-blast exposure: molecular, histopathological, and behavioral consequences. Sci. Rep. 10, 17446.

50. Armstrong, R.C., Mierzwa, A.J., Marion, C.M., and Sullivan, G.M. (2016) White matter involvement after TBI: clues to axon and myelin repair capacity. Exp. Neurol. 275, Pt. 3, 328-333.

51. Marion, C.M., McDaniel, D.P., and Armstrong, R.C. (2019). Sarm1 deletion reduces axon damage, demyelination, and white matter atrophy after experimental traumatic brain injury. Exp. Neurol. 321, 113040.
Cite this article as: Bradshaw Jr, DV, Kim, Y, Fu, A, Marion, CM, Radomski, KL, McCabe, JT, and Armstrong, RC (2021) Repetitive blast exposure produces white matter axon damage without subsequent myelin remodeling: in vivo analysis of brain injury using fluorescent reporter mice. Neurotrauma Reports 2:1, 180-192, DOI:10.1089/ neur.2020.0058

$\begin{aligned} & \quad \text { Abbreviations Used } \\ & 3 \mathrm{D}=\text { three-dimensional } \\ & \mathrm{ABS}=\text { Advanced Blast Simulator } \\ & \mathrm{ANOVA}=\text { analysis of variance } \\ & \mathrm{CC}=\text { corpus callosum } \\ & \mathrm{DAPI}=4^{\prime}, 6 \text {-diamidino-2-phenylindole } \\ & \mathrm{DTI}=\text { diffusion tensor imaging } \\ & \mathrm{GFAP}=\text { glial fibrillary acidic protein } \\ & \mathrm{GFP}=\text { green fluorescent protein } \\ & \mathrm{IBA} 1=\text { ionized calcium binding adaptor molecule } 1 \\ & \mathrm{mG}=\text { membrane-localized GFP } \\ & \mathrm{MOG}=\text { myelin oligodendrocyte glycoprotein } \\ & \mathrm{MRI}=\text { magnetic resonance imaging } \\ & \mathrm{mT}=\text { tdTomato } \\ & \mathrm{OPC}=\text { oligodendrocyte progenitor cell } \\ & \mathrm{ROI}=\text { region of interest } \\ & \mathrm{SEM}=\text { standard error of the mean } \\ & \mathrm{TBI}=\text { traumatic brain injury } \\ & \mathrm{TMX}=\text { tamoxifen } \\ & \mathrm{YFP}=\text { yellow fluorescent protein } \\ & \beta-\mathrm{APP}=\beta \text {-amyloid precursor protein }\end{aligned}$

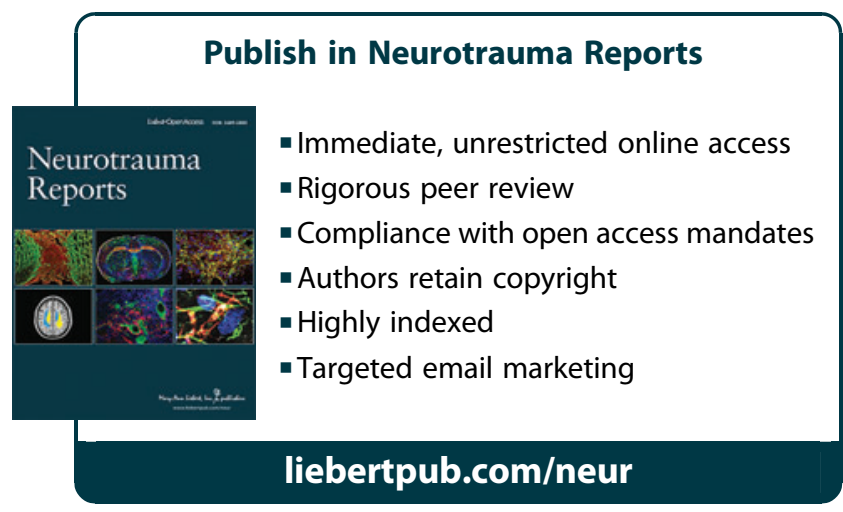

